\title{
High-Throughput Nuclear Magnetic Resonance Metabolomic Footprinting for Tissue Engineering
}

\author{
CHRISTOPHER SEAGLE, Ph.D., ${ }^{1}$ MEGAN A. CHRISTIE, M.S., ${ }^{2}$ JASON H. WINNIKE, M.S., ${ }^{1}$ \\ RANDALL E. MCCLELLAND, Ph.D., JOHN W. LUDLOW, Ph.D., ${ }^{3}$ THOMAS M. O'CONNELL, Ph.D., \\ MICHAEL P. GAMCSIK, Ph.D., ${ }^{1}$ and JEFFREY M. MACDONALD, Ph.D. ${ }^{1}$
}

\begin{abstract}
We report a high-throughput (HTP) nuclear magnetic resonance (NMR) method for analysis of media components and a metabolic schematic to help easily interpret the data. Spin-lattice relaxation values and concentrations were measured for 19 components and 2 internal referencing agents in pure and 2-day conditioned, hormonally defined media from a 3-dimensional (3D) multicoaxial human bioartificial liver (BAL). The ${ }^{1} \mathrm{H}$ NMR spectral signal-to-noise ratio is 21 for $0.16 \mathrm{mM}$ alanine in medium and is obtained in 12 min using a $400 \mathrm{MHz}$ NMR spectrometer. For comparison, 2D gel cultures and 3D multicoaxial BALs were batch cultured, with medium changed every day for 15 days after inoculation with human liver cells in Matrigel-collagen type 1 gels. Glutamine consumption was higher by day 8 in the BAL than in 2D culture; lactate production was lower through the 15-day culture period. Alanine was the primary amino acid produced and tracked with lactate or urea production. Glucose and pyruvate consumption were similar in the BAL and 2D cultures. NMR analysis permits quality assurance of the bioreactor by identifying contaminants. Ethanol was observed because of a bioreactor membrane "wetting" procedure. A biochemical scheme is presented illustrating bioreactor metabolomic footprint results and demonstrating how this can be translated to modify bioreactor operational parameters or quality assurance issues.
\end{abstract}

\section{INTRODUCTION}

$\mathbf{H}$ IGH-THROUGHPUT (HTP) nuclear magnetic resonance (NMR) spectroscopic analysis of media provides an unbiased determination of biochemical concentrations, permitting culture protocol modifications to be implemented rapidly for optimizing culture conditions. ${ }^{1-3}$ In combination with multivariate statistical methods, ${ }^{4}$ this analytical method provides robust characterization of phenotypes for tissue engineering. Unlike other analytical methods such as mass spectrometry, NMR spectroscopy does not require extensive sample preparation and is innately quantitative, resulting in unbiased analyses. This makes NMR spectroscopy one of the most powerful analytical techniques available for metabolic profiling. The global profiles are also valuable from a quality control perspective, which is particularly important for tissue engineering because of the multitude of operational parameters and potential for human error and contamination. This is especially true for engineered bioartificial liver (BAL), in which clinical liver assays are used to characterize degrees of liver function and differentiation. There is a need for a more-robust method of reporting the hepatic phenotype of BALs. ${ }^{5}$ Metabolomic analysis fills this requirement. ${ }^{6}$

The state-of-the-art method to quantify concentrations in media is high-performance liquid chromatography coupled with mass spectrometry. ${ }^{7-9}$ Media component analysis

\footnotetext{
${ }^{1}$ Department of Biomedical Engineering, University of North Carolina at Chapel Hill, North Carolina.

${ }^{2}$ ADMET Technologies Inc., Durham, North Carolina.

${ }^{3}$ Tengion Inc., Winston-Salem, North Carolina.

${ }^{4}$ Division of Pharmacotherapy and Experimental Therapeutics, School of Pharmacy, University of North Carolina at Chapel Hill, Chapel Hill, North Carolina.
} 
using ${ }^{1} \mathrm{H}$ NMR spectroscopy has been attempted since the $1980 \mathrm{~s}^{1-3,10-12}$ but has never been widely accepted, probably because of the lower sensitivity of NMR. Now with high-field NMR spectrometers more common and development of multivariate statistics for metabolomics, ${ }^{13,14}$ the unbiased nature of NMR makes it perhaps one of the most useful techniques for phenotyping tissues, especially for 3-dimensional (3D) tissue constructs in tissue engineering, because it simultaneously provides quality assurance. Although direct analysis of the tissue can be performed, the bioreactor dimensions must conform to existing NMR hardware ${ }^{15}$ or the NMR hardware must be built around the bioreactor $^{16}$ in a similar fashion as the NMR/magnetic resonance imaging analysis of human tissues. Medium analysis serves a similar function as analyzing the central compartment, or blood, of a human. Two different metabolic profiles provide the distinctions between the compliment of metabolites within the cells_- "the fingerprint" - and from those outside the cells_- "the footprint." 17 The fingerprint and footprint are tissue specific and depend on the degree of metabolic differentiation, resulting from the number and capacity of the various transporter, signaling, and enzymatic pathways.

The same problems associated with NMR spectroscopic analysis of serum apply to culture media, including differences in spin-lattice relaxation $\left(T_{1}\right)$ values due to nonspecific protein binding resulting in inaccurate determination of concentrations. ${ }^{18}$ The $T_{1}$ values have not been reported for components in culture media and are necessary to ensure accuracy of the NMR-derived concentrations. ${ }^{3}$ Therefore, a HTP NMR method has been optimized considering the $T_{1}$ values of medium components. In addition, the sample preparation conditions were optimized and the spectral parameters identified to obtain ${ }^{1} \mathrm{H}$ NMR spectra of media within 12 min and using spectral integration to easily convert peak areas to metabolite concentrations. To demonstrate the application of the HTP NMR method to optimizing tissue culture protocol, we applied the method to development of a commercially available BAL. ${ }^{19,20}$

\section{METHODS}

\section{Materials}

NMR supplies were purchased from multiple vendors. The 5-mm tubes, alanine, glutamic acid, glucose, valine, methionine, sodium citrate, and imidazole were purchased from Sigma Chemical company (St. Louis, MO). Deuterium oxide, sodium formate, and 3-(trimethylsily)propionic2,2,3,3- $\mathrm{d}_{4}$ acid were purchased from Cambridge Isotope Laboratories (Andover, MA), Alfa Aesar (Ward Hill, MA), and Isotec Inc. (Miamisburg, $\mathrm{OH}$ ), respectively. Bioreactor end pieces were machined from polypropylene tube (1" diameter) and assembled as previously described. ${ }^{19}$ Bioreactor life support units have been described previously. ${ }^{16}$
Matrix supporting the cells was made up of 1:1 (vol:vol) collagen type I and Matrigel from Inamed Biomaterials Inc. (Irvine, CA) and Becton Dickinson Inc. (Franklin Lakes, NJ).

\section{$B A L$ inoculation, medium, and medium collection}

The human 3D BAL studies were performed at the ADMET Technologies Inc. (Research Triangle Park, NC) laboratory using their patented multicoaxial bioreactor ${ }^{21}$ and their primary human liver cells, UMIX, as described on their Web page (http://www.admettechnologies.com/). Liver cells were mixed with a 1:1 collagen-to-Matrigel mixture (vol:vol) and inoculated at a density of $10^{7}$ cells $/ \mathrm{mL}$. The medium was a hormonally defined recipe, Kubotas media $^{22}$ using Dulbecco's modified Eagle medium as the basal medium (Mediatech 17-205-CV, Herndon, VA). The medium was changed in batch mode the day after inoculation and then every 2 days thereafter and placed in a $80^{\circ} \mathrm{C}$ freezer until analysis, at which point it was thawed. Flow rates through the $\mathrm{BAL}$ were $5 \mathrm{~mL} / \mathrm{min}$, and oxygen concentration was $95 \%$, as described by McClelland and Coger. $^{23}$

\section{D Culture of UMIX}

Hepatocytes obtained from a single human liver were cultured immediately after isolation using the same cell and matrix mix as described in the above "methods" section, was plated on a precoated 6-well collagen type I plate (Becton Dickinson, Franklin Lakes, NJ) at a $1.25-\mathrm{mL}$ volume per well, or 12.5 million cells. The cell and matrix solution was placed in a $37^{\circ} \mathrm{C}$ humidified incubator with $5 \%$ carbon dioxide for $1 \mathrm{~h}$ to gel. Medium was then added to the culture at a volume of $2 \mathrm{~mL}$ per well. Medium was changed $24 \mathrm{~h}$ after plating and every other day thereafter for all cultures.

\section{$3 D$ Culture of UMIX}

Figure 1 shows the multicoaxial bioreactor with all of the ports identified (left) and the bioreactor incorporated into the life support system (right). The bioreactor is placed inside an incubator with the reservoir, pump, and various tubings. The gas is composed of oxygen:carbon dioxide (95:5), and the gas line is inserted through the side of the incubator and attached directly to the bioreactor, dead-ending at the reservoir, which vents through a $0.2-\mu \mathrm{m}$-pore polypropylene air filter. The temperature of the incubator is $37^{\circ} \mathrm{C}$.

\section{Optimization of NMR pulse sequence for quantification of medium components}

Four pulse sequences were compared for maximum signal-to-noise ratio, the ${ }^{1} \mathrm{H}$ 1-pulse with presaturation using $45^{\circ}$ and $90^{\circ}$ flip angles with delay of 5 -fold $\mathrm{T}_{1}$, a $\mathrm{T}_{2}$-weighted Carr-Purcell-Meiboom-Gill (CPMG), and a 


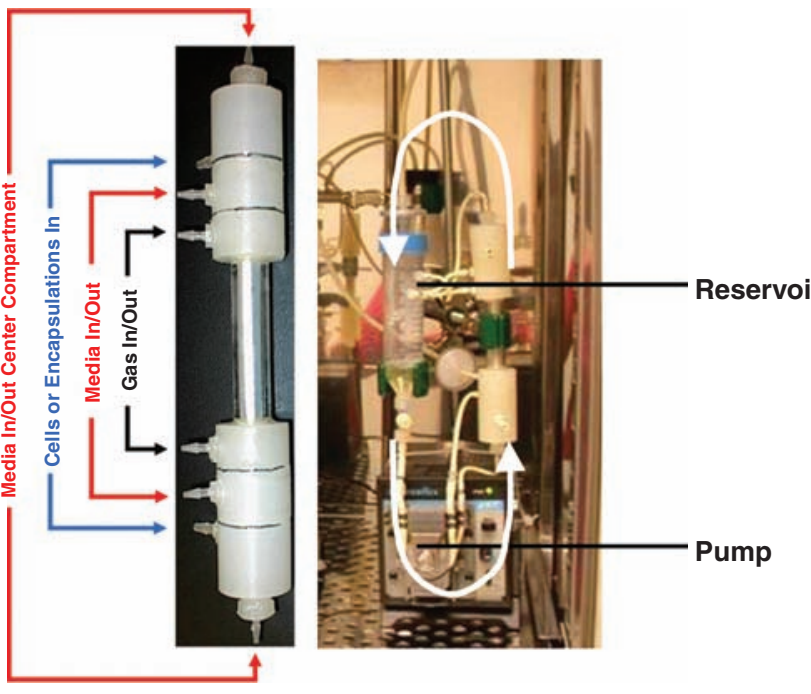

FIG. 1. Bioreactor (left) and its incorporation into the life-support loop (right). Color images available online at www.liebertpub.com/ ten.

$\mathrm{T}_{1}$-weighted 1D nuclear Overhauser effect spectroscopy (NOESY). A 1-pulse sequence with a $4 \mathrm{~s}$ presaturation pulse was used with a sweep width of $4406.2 \mathrm{~Hz}$ and $32 \mathrm{~K}$ complex data points, resulting in an acquisition time of $3.72 \mathrm{~s}$. The 1 -pulse sequence used a $45^{\circ}$ or a $90^{\circ}$ flip angle with a $18.88 \mathrm{~s}$ or a $41.28 \mathrm{~s}$ interpulse delay and a total repetition time of $22.5 \mathrm{~s}$ or $45 \mathrm{~s}$, respectively. A total of 32 transients were obtained, resulting in 24 min per spectrum. For accurate comparison, the same parameters used for the 1-pulse sequence were used for the CPMG and 1D NOESY, with addition of a 40-ms and 100-ms interpulse delay and mixing time, respectively.

For determining $T_{1}$ values for multiple medium components and internal standards, an inversion recovery sequence was used, with the longest interpulse delay of $60 \mathrm{~s}$. One pure medium sample and one "conditioned" medium sample were prepared with $8.15 \mathrm{mM}$ formate, $25 \mathrm{mM}$ citrate, $143 \mathrm{mM}$ imidazole, and $890 \mu \mathrm{M}$ 3-trimethylsilyl[2,2,3,3D4]-propionate (TSP). The longest $\mathrm{T}_{1}$ value was $8.3 \mathrm{~s}$ for the concentration standard formate; this value was used to calculate repetition times to ensure that fully relaxed spectra were obtained. These compounds were used to observe effects of $T_{1}$ and $T_{2}$ on peak areas, whereas citrate and imidazole served to measure paramagnetic cation concentrations ${ }^{24}$ and $\mathrm{pH}$, respectively. Sensitivity comparisons of the various pulse sequences were made by calculating the signal-to-noise ratio using 1D NMR Processor 8.0 (Advanced Chemistry Development, Toronto, Ontario, CA).

\section{${ }^{I} H$ NMR analysis of medium}

Samples were made from $540 \mu \mathrm{L}$ of medium and $60 \mu \mathrm{L}$ of deuterium oxide containing $8.9 \mathrm{mM}$ TSP and $81.5 \mathrm{mM}$ formate (final sample concentrations $890 \mu \mathrm{M}$ and $8.15 \mathrm{mM}$, respectively). Because TSP binds to proteins, resulting in significant peak broadening ${ }^{18}$, formate was added as a second concentration standard to validate the effect of TSP binding to albumin in the media. A 1-pulse sequence was used with a $90^{\circ}$ flip angle and 45 -s repetition time, using the parameters described above. To minimize NMR analysis and create a HTP analysis, 16 transients resulted in a sufficient signal-to-noise ratio for the 19 peaks measured in the medium, resulting in 12-min spectra. Mean signal-tonoise for 16 randomly selected samples taken from the $\alpha$-C1-spectral set was determined to be $136.74 \quad 43.57$ for the glucose peak at $5.25 \mathrm{ppm}$, which represents a range of concentration for the samples of between 12 and $20 \mathrm{mmol}$. In combination with $3 \mathrm{~min}$ for robotic sample changing and gradient shimming, each sample can be analyzed in $15 \mathrm{~min}$. The free induction decays (FIDs) were processed with a $0.5 \mathrm{~Hz}$ exponential, zero-filled to $64 \mathrm{~K}$ data points, and Fourier transformed into the frequency domain.

Absolute sample concentrations were determined using area under curve (AUC) for selected metabolite peaks normalized to formate concentration and adjusted for the proton number associated with the peak of interest. Consumption and production rates were calculated as the millimolar difference between the 2-day medium changes divided by the number of remaining viable hepatocytes inoculated in the BAL or $2 \mathrm{D}$ culture dish per hour. Evaporation differences were corrected by normalizing formate concentrations and applying correction factors to all constituents within each individual 2-day sample.

\section{Validation and accuracy of the HTP NMR method}

To validate the NMR method and determine the accuracy, ${ }^{1} \mathrm{H}$ NMR quantification of 5 analytes were compared with gravimetric analysis using a balance Mettler Toledo XS205 (Mettler Toledo Inc., Columbus, OH). The accuracy of the balance was used in determining the accuracy of the gravimetric method. The NMR standards (TSP, imidazole, and formate) and analytes (alanine, glutamic acid, glucose, valine, and methionine) were lyophilized overnight to eliminate moisture, which is particularly common in the southeastern United States. Concentrations at $100 x$ the medium recipe for alanine $(5 \mathrm{mM})$, glutamic acid $(1.3 \mathrm{M})$, glucose $(1.9 \mathrm{M})$, valine $(45.2 \mathrm{mM})$, and methionine $(11.6 \mathrm{mM})$ were made in $10-\mathrm{mL}$ volumetric flasks. These analytes were chosen because they gave a range of concentrations and spectral resolution. Two samples of 2-day conditioned medium obtained on day 11 of culture from the 2D and 3D cultures were used for the study. Sample 1 was used to determine the amount of formate in the $2 \mathrm{D}$ and $3 \mathrm{D}$ media, and it was composed of $540 \mu \mathrm{L}$ of medium and $60 \mu \mathrm{L}$ of deuterium oxide containing $8.9 \mathrm{mM}$ TSP and $81.5 \mathrm{mM}$ imidazole. To determine the accuracy of the NMR method, Sample 2 was composed of $400 \mu \mathrm{L}$ of sample 1, $80 \mu \mathrm{L}$ of deuterium oxide, $60 \mu \mathrm{L}$ of standard solution containing $8.9 \mathrm{mM}$ TSP, $81.5 \mathrm{mM}$ imidazole, and $81.5 \mathrm{mM}$ 
formate, and $12 \mu \mathrm{L}$ of each of the five $100 \times$ analyte solutions. The percent error for the NMR method was calculated for each of the analytes by determining the concentration in Sample 2 and subtracting the respective stock media analyte concentration obtained from Sample 1 multiplied by the dilution factor, 0.66 , and divided by the theoretical concentration derived from the gravimetric measurement of known additions. Formate in Sample 1 was used to check for potential TSP AUC affects due to protein binding between the 2 samples.

\section{NMR peak area determination}

A macro was written to process the FIDs using $0.5 \mathrm{H}_{\mathrm{z}}$ Lorentzian apodization and baseline corrected using the multipoint baseline correction option. Quantitative analysis of the peaks was carried out using 2 methods implemented in the ACD software. First, the peaks were fitted using the peak deconvolution with Lorentzian lineshapes. The second method used the intelligent binning integration protocol. The known concentration of TSP and formate were used as internal concentration standards.

\section{Viability and functional assays}

Media supernatants were analyzed at 48-hour intervals to monitor liver functions, specifically lactate dehydrogenase (LDH) leakage and urea production. To determine the number of live cells remaining in culture, $\mathrm{LDH}$ was quantified using a Vitros DT60 II chemistry system from Johnson \& Johnson (New Brunswick, NJ). A standard curve was produced using a known number of cells in a $2-\mathrm{mL}$ volume of medium, sonicating the cells, and measuring the LDH. Urea production was determined using a diagnostic kit purchased from BioAssay Systems (Hayward, CA). Briefly, a chromogenic reagent forms a colored complex specifically with urea, and the color is then measured at $520 \mathrm{~nm}$ using a BioTek microplate reader (Winooski, VT).

\section{Metabolomic footprint and the empirical mass balance model}

Metabolic pathway reconstruction was done by entering medium components into the KEGG or Brenda metabolomic databases (http://www.genome.jp/kegg/) generating connecting pathways. ${ }^{4}$ Consumption and production rates were entered into the biochemical mass balance schematic representation of the metabolomic footprint. The metabolomic footprint was obtained over 15 days of culture, and different metabolic states were defined as a shift in the preferred carbon source from glucose to glutamine with a concomitant decrease in production of lactate, alanine, or both. This process of distilling quantitative changes in consumption and production rates to a simple biochemical schematic is the empiric model, which can be used to optimize operational parameters.

\section{Microscopy techniques}

The morphology of the cells in 2D cultures were examined using a Leica DMIL inverted phase-contrast microscope (Leica Microsystems GMbH, Wetzlar, Germany). Pictures were taken every other day, coinciding with medium changes. The BAL cultures were imaged as previously described. ${ }^{23}$

\section{RESULTS}

Figure 2 is a ${ }^{1} \mathrm{H}$ NMR spectrum of the culture medium (middle) showing the various peaks representing compounds found in the medium. (See Fig. 2A legend for assignments.) The $T_{1}$ values of the various peaks in pure medium are shown in the plot on the top of Figure 2B. In general, the conditioned medium had lower $\mathrm{T}_{1}$ values; this difference in $\mathrm{T}_{1}$ values is shown at the bottom of the plot in Figure 2B. There is broadening of the TSP NMR signal that is likely due to the albumin concentration, because albumin binds TSP, decreasing its spin-spin relaxation $\left(\mathrm{T}_{2}\right)$ value. ${ }^{18}$ Therefore, formate was used as a concentration standard, because it has been show not to be involved in nonspecific binding. ${ }^{18}$ That the $\mathrm{T}_{1}$ value did not change with the addition of albumin confirmed this. However, the $\mathrm{T}_{1}$ value of formate in conditioned media decreases (Fig. 2B), indicating that something created by the hepatocytes or extracellular matrix caused this decrease.

Further comparison of ${ }^{1} \mathrm{H}$ presaturation 1-pulse, 1D NOESY ( $\mathrm{T}_{1}$-weighted) and CPMG $\left(\mathrm{T}_{2}\right.$-weighted) resulted in similar values for peak areas using integration or deconvolution. As expected from the $\mathrm{T}_{1}$ values shown in Figure 2B, the $\mathrm{T}_{1}$-weighted 1D NOESY decreased the TSP peak area. (See Methods for sequence parameters.) Overall, ${ }^{1} \mathrm{H}$ presaturation 1 -pulse spectra with a $45^{\circ}$ or $90^{\circ}$ flip angle had the same signal-to-noise ratio for a given time. Because the hormonally defined or serum-supplemented medium contains one-tenth of the albumin as normal serum, the dramatic effects on TSP peak width observed with serum ${ }^{18}$ were not seen with culture medium. The $\mathrm{T}_{2}$-weighted CPMG did not affect the quantification of TSP, and no detectable paramagnetic load was observed, as measured from citrate broadening. However, because of a potential effect on $\mathrm{T}_{2}$ values caused by, for example, massive hepatocyte death causing increased iron load, the simple 1pulse spectrum was chosen as the optimum pulse sequence. Therefore, a simple ${ }^{1} \mathrm{H}$ presaturation 1-pulse with $90^{\circ}$ flip angle and 45 -s repetition time ( 5 times the longest $\mathrm{T}_{1}$ value) was determined to be the most-quantitative pulse sequence. Comparison of peak fitting (i.e., deconvolution) and manual integration resulted in less than $1 \%$ difference in concentration measured using well-resolved peaks.

Figure 3 shows portions of two ${ }^{1} \mathrm{H}$ NMR spectra of medium from 2D cultures with just TSP and imidazole added (top) and formate, glucose, methionine, glutamic acid, alanine, and valine added (bottom). The aliphatic portion of 

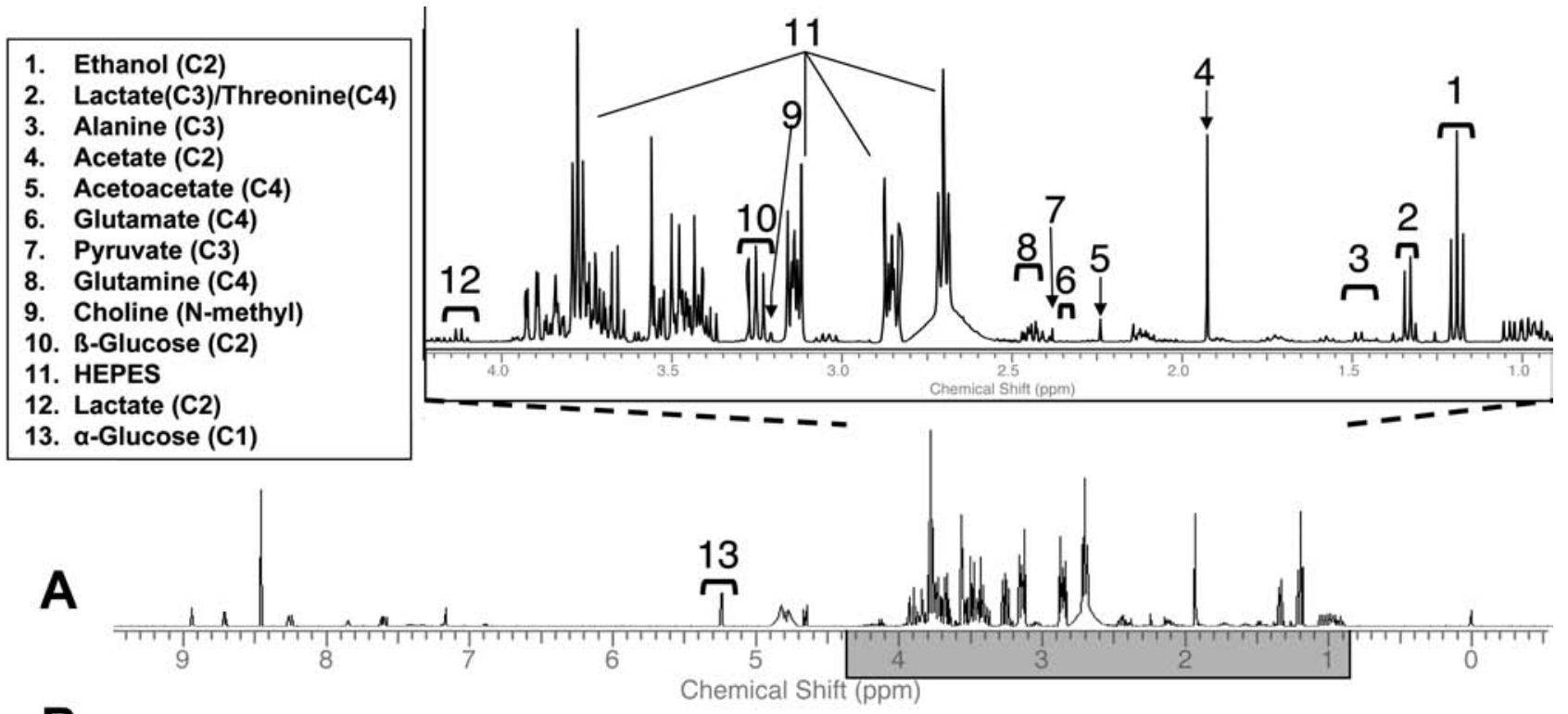

B

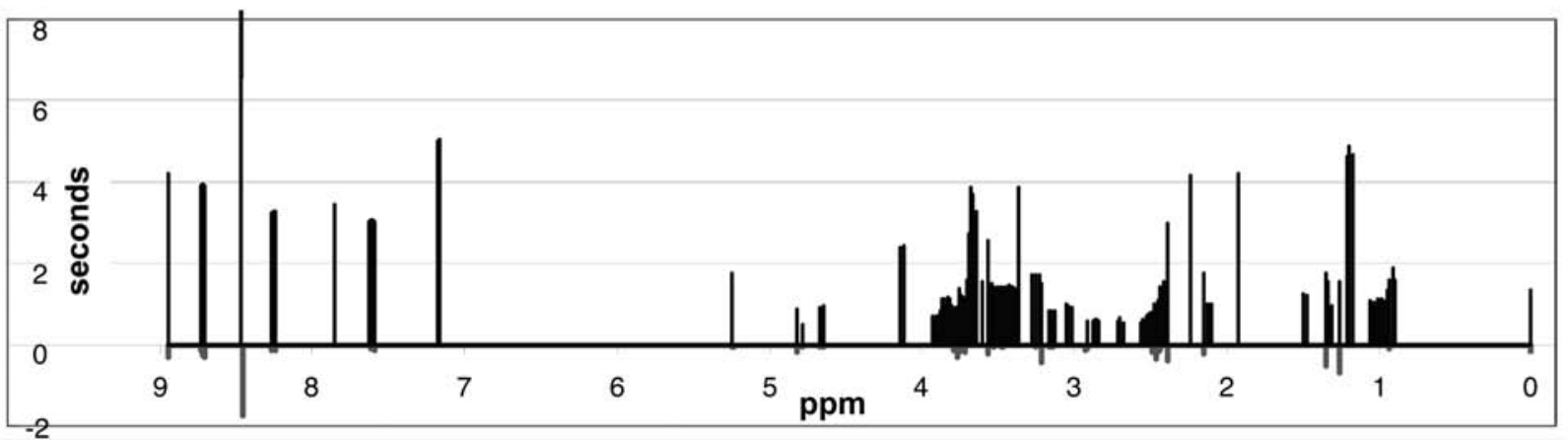

FIG. 2. ${ }^{1} \mathrm{H}$ nuclear magnetic resonance spectra of medium exposed to bioreactor-containing cells (A), T1 (above) and delta T1 (below) values for fresh bioreactor medium and cell-exposed bioreactor media as a function of chemical shift (B). Assignments are inset.

the ${ }^{1} \mathrm{H}$ NMR spectrum in Figure 3 (bottom spectrum) demonstrates that there are nearly undetectable levels of formate in the $2 \mathrm{D}$ media, and the $3 \mathrm{D}$ culture medium showed the same results. Figure 3 (bottom) is the same 2D medium sample shown in Figure 3 (top) but with known amounts of formate and the 5 analytes as described in the Methods section. In comparison with the theoretical values obtained from gravimetric measurement of the added analytes, glucose, methionine, glutamic acid, alanine, and valine had $1.8 \%, 6.6 \%, 4.6 \%, 2.2 \%$, and $0.8 \%$ errors, respectively, as described in the Methods section. The broad peak down field (to the left) of glutamic acid has shifted down field. This peak is due to N-2-hydroxyethylpiperazine-N'-2ethanesulfonic acid (HEPES) and shifts with $\mathrm{pH}$. A pH less than 6.5 is required to obtain a sufficiently flat baseline to obtain accurate measures of glutamine, which in this case is the reason for the negative error of this resonance. Glutamine $(\mathrm{C} 4,2.45 \mathrm{ppm})$ is also hampered by resonances up field, such as $\mathrm{C} 3$-pyruvate $(2.36 \mathrm{ppm})$ and the multiplet of C4-glutamate $(2.35 \mathrm{ppm})$, so setting the integration just inside the outermost up-field part of the multiplet also underestimates C4-glutamine. Methionine (2.14 ppm) co-resonates with $\mathrm{C} 3$-glutamine $(2.13 \mathrm{ppm})$ and does not demonstrate sufficient peak separation at this field strength to be quantified.

One interesting finding demonstrating the inherent unbiased quantification of NMR spectroscopy was the serendipitous discovery of a dilution factor in the bioreactor medium from the first medium change, which the phosphate buffered saline (PBS) rinse procedure caused. As described in the Methods session, the bioreactor membrane was wetted with ethanol and then rinsed 2 times with PBS and once with medium before a final medium change prior to inoculation. The use of concentration standards immediately demonstrated an overall decrease of medium component concentrations.

Figure 4 shows calculated viable cells according to values of the cumulative medium $\mathrm{LDH}$, a measure of mortality, over the course of culture (15 days). In general, LDH activity was slightly higher in $3 \mathrm{D}$ BAL than in the $2 \mathrm{D}$ 


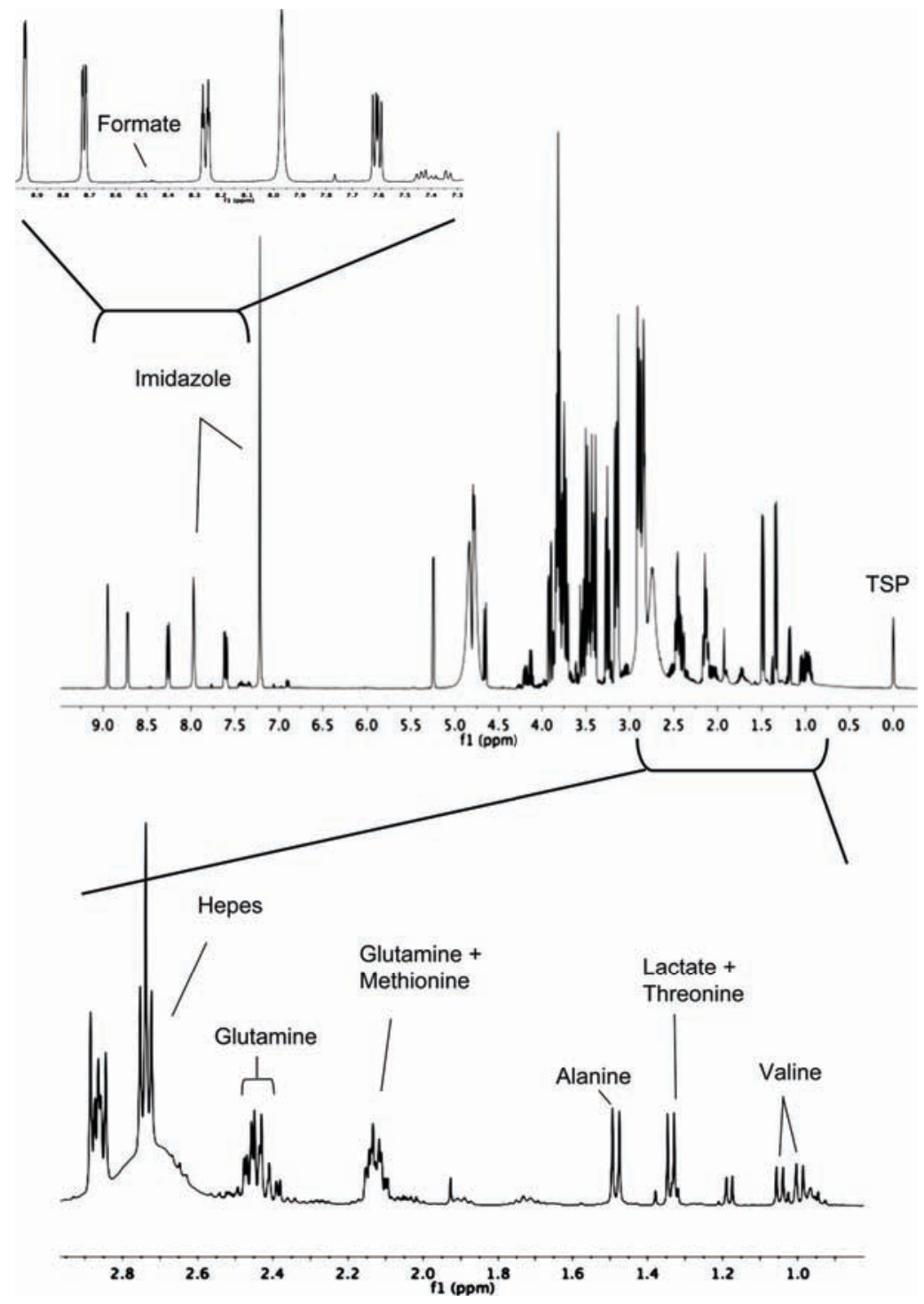

FIG. 3. ${ }^{1} \mathrm{H}$ nuclear magnetic resonance spectra of media from 2-dimensional cultures obtained from days 9 to 11 of culture with addition of 3-trimethylsilyl[2,2,3,3-D4]-propionate (TSP) and imidazole (top) and the addition of formate, glucose alanine, glutamic acid, methionine, and valine (bottom) as concentrations given in the Methods. Inset on top is an enlargement demonstrating nearly undetectable formate. HEPES, N-2-hydroxyethylpiperazine-N'-2-ethanesulfonic acid.

control culture. By measuring the LDH level of 7 aliquots of hepatocytes obtained from the same isolation used in the bioreactor experiments ranging in cell density from 65,000 cells $/ \mathrm{mL}$ to $4.16 \times 10^{6}$ cells $/ \mathrm{mL}$, a calibration line was developed to calculate the cell death for each 48-hour medium change (Fig. 5). The summation of these LDH values was taken and used to determine the total viable cell number (Fig. 4B) and the percentage cumulative cell death (Fig. 4A) based upon the equation determined according to the calibration plot (Fig. 5) and plotted for $2 \mathrm{D}$ and $3 \mathrm{D}$ culture (Fig. 4A). These mortality values were used to calculate the consumption and production rates of the $2 \mathrm{D}$ and 3D culture as a function of viable cells rather than initial inoculation number. The primary difference between
2D and 3D cultures, other than dimensionality, was oxygen concentration, with $20 \%$ and $95 \%$ oxygen-gas mixture, respectively.

Figure 6 displays the time courses of consumption rates of the major nutrients (glucose (Fig. 6A), pyruvate (Fig. 6B), and glutamine (Fig. 6C)) and the lactate production rate (Fig. 6D) for the 3D BAL and 2D control cultures. The glucose and pyruvate consumption rates were fairly similar in 2D and 3D culture systems throughout the 15 days of culture. Of particular interest is the difference between glutamine consumption rates and lactate production rates, because aerobic and anaerobic metabolism consume and produce, respectively, these nutrients. Glutamine enters directly into the TCA cycle as $\alpha$-ketoglutarate. The 3D BAL 
A

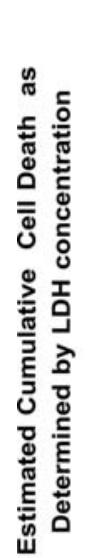

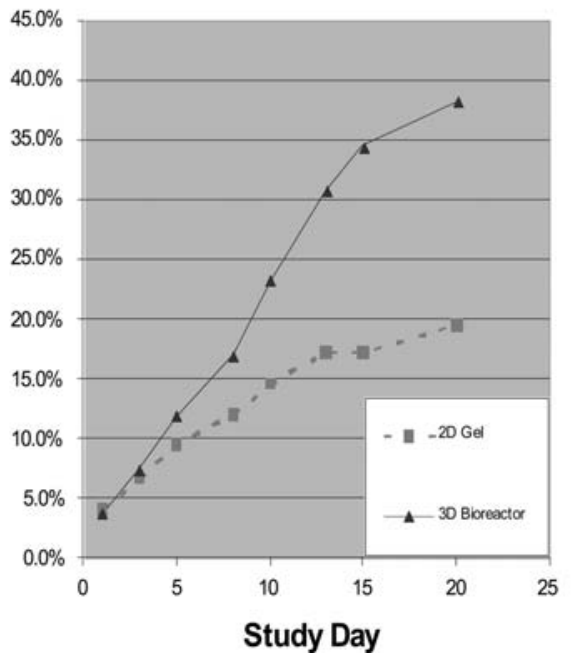

B

\begin{tabular}{|ccc|}
\hline \multicolumn{3}{|c|}{$\begin{array}{c}\text { Estimated Live Cell } \\
\text { Count (millions) }\end{array}$} \\
\multicolumn{4}{|c|}{$\begin{array}{cc}\text { Study } \\
\text { Day }\end{array}$} & 2D Gel & 3D \\
0 & & Bioreactor \\
\cline { 3 - 3 } 1 & 12.5 & 12.5 \\
3 & 11.99 & 12.04 \\
5 & 11.66 & 11.56 \\
7 & 11.31 & 11.01 \\
9 & 11.00 & 10.38 \\
11 & 10.67 & 9.60 \\
13 & 10.35 & 8.66 \\
15 & 10.35 & 8.19 \\
& 10.06 & 7.73 \\
\hline
\end{tabular}

FIG. 4. Estimated cumulative cell death as a function of study day (A), and estimated viable cells (B).

ceases to produce lactate by day 5, while continuously consuming glutamine throughout the culture period, indicating a shift to aerobic metabolism, with the TCA cycle dominating energy production. Alternatively, the 2D control has increasing production of lactate, demonstrating that the majority of the hepatocytes rely on anaerobic glycolysis.

Figure 7 are the urea (Fig. 7A) and alanine (Fig. 7B) production rates in $3 \mathrm{D} \mathrm{BAL}$ and $2 \mathrm{D}$ control cultures. Alanine production tracks urea production and will generate ammonia for the urea cycle. ${ }^{25}$ After day 8 of culture, alanine is consumed. Alanine also tracks lactate production and is produced in conjunction with lactate during anaerobic glycolysis via transamination of pyruvate with glutamate. ${ }^{25}$

Figure 8 are the ethanol concentration (Fig. 8A) and acetate production (Fig. 8B) time courses for the 3D BAL and 2D control cultures. Ethanol is discovered in the 3D bioreactor via the membrane wetting procedure described in the Methods section. The middle hollow fiber is made of polypropylene and requires ethanol wetting to induce water permeability. There should be additional rinsing steps with PBS to wash out re-

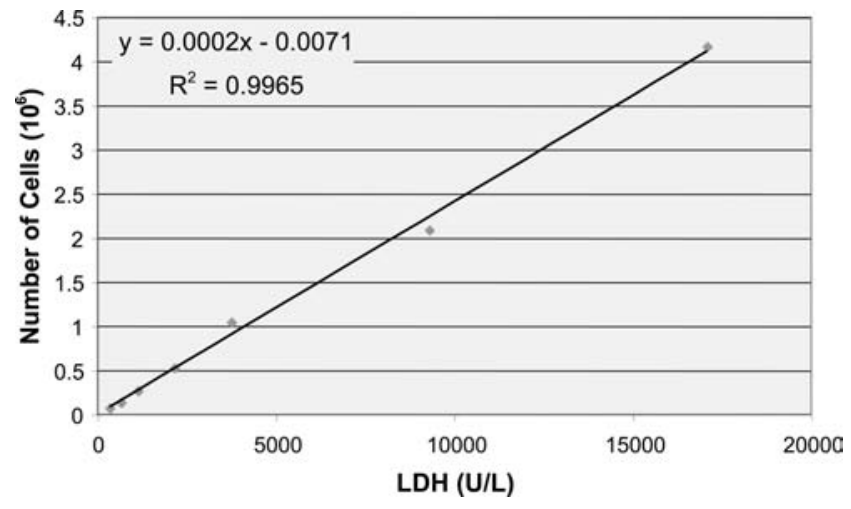

FIG. 5. Lactate dehydrogenase calibration curve. sidual ethanol. The hepatocytes actively metabolize the ethanol to acetate using alcohol dehydrogenase and aldehyde dehydrogenase. There is a spike in acetate levels by day 7 in 2D control cultures. This could be due to ethanol metabolism, but the significantly higher acetate production rate in $2 \mathrm{D}$ cultures $\left(0.6 \mathrm{mM} / 10^{6}\right.$ cells/hr) versus $3 \mathrm{D}$ BAL cultures $\left(0.05 \mathrm{mM} / 10^{6}\right.$ cells $\left./ \mathrm{hr}\right)$ even in the absence of detectable ethanol (Fig. 8) suggests additional processes at work. By day 3 of 2D cultures, any acetate leaching from the collagen; Matrigel $^{\mathrm{TM}}$ had subsided to nearly undetectable levels (Fig. 8B). Under normal physiological conditions, acetate accumulates during fasting because of the $\beta$-oxidation of lipids, resulting in 2 carbon units of acetate. The various other amino acids and nicotinamide did not change significantly over the course of the experiment.

\section{DISCUSSION}

Although previous NMR "footprinting" studies of cell culture have been performed, $1,3,8,10-12,17$ this is the first example demonstrating the HTP, unbiased nature of NMR spectroscopic analysis especially for tissue engineering. Peak overlap of compounds can introduce errors that arise when concentration measurements are performed on spectra that may include contributions within a single visible peak that arise from 2 peaks superimposed on each other. Therefore, it is always necessary to check the binned regions of each spectrum to ensure that no contamination from undesired resonances occurs. Most metabolites display multiple peaks in different regions of the spectrum, allowing for selection of peaks, which can be used to determine concentration without the challenge associated with co-resonance. Two examples shown in Figure 3 are methionine, of which it was not possible to obtain accurate measurements because of co-resonance with $\mathrm{C} 3$-glutamine. 

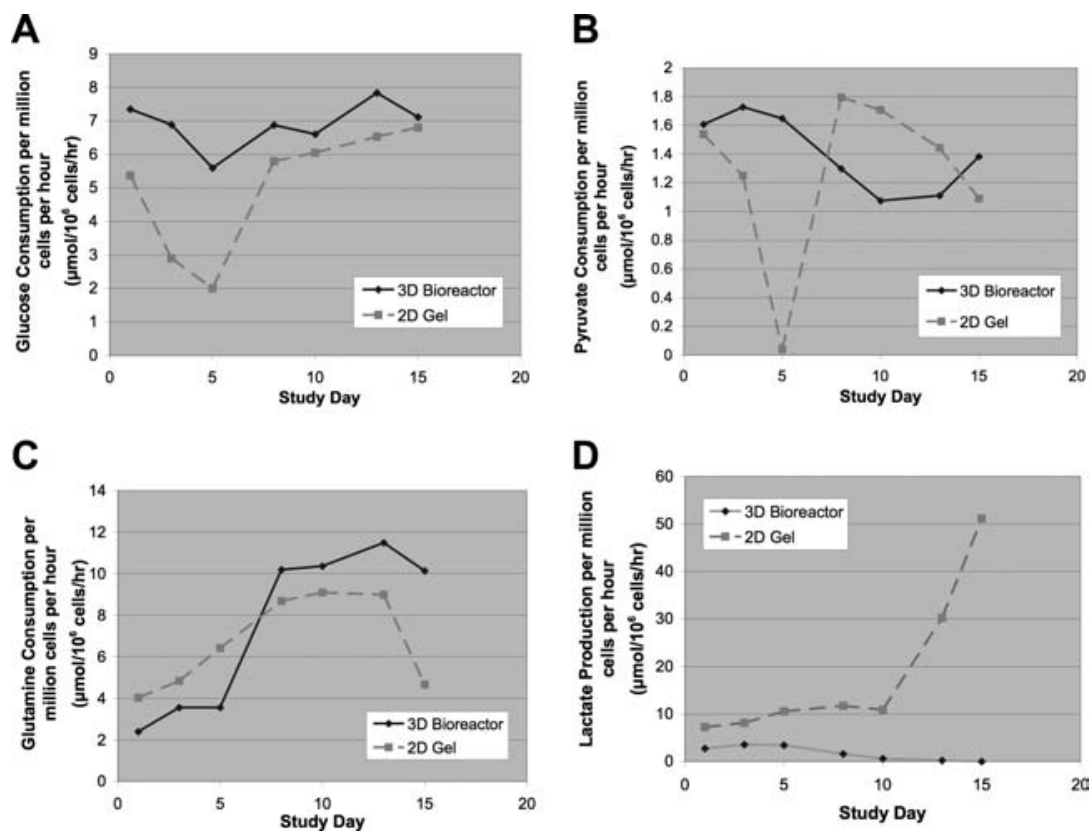

FIG. 6. Time courses of glucose, pyruvate, and glutamine consumption (A-C) and lactate production (D) in 3D bioartificial liver and 2D control cultures.

Alternatively C4-glutamine can be resolved if a sample $\mathrm{pH}$ less than 6.5 is used to resolve the down-field broad component due to HEPES, which affects the baseline. In addition, careful placement of the up-field integral boundary is important, otherwise, part of the multiplet gets clipped, underestimating the measurement.

Sample contamination, macromolecular content, $\mathrm{pH}$, and paramagnetic strength causing perturbations on peak area and chemical shifts may also challenge accurate concentration measures. ${ }^{26}$ These multivariate aspects of quantitative NMR makes the choice of a concentration standard difficult. A recent study analyzing media from a human cell-line, HepG2, showed the presence of significant amounts of formate, which was used as a concentration standard in the present study. ${ }^{11}$ Formate was not detected in the medium samples from our 2D and 3D primary human liver cultures (Fig. 3). This could be due to their addition of serum to their medium which contains formate, upregulation of 1-carbon metabolism in the transformed human HepG2 cell line, or the extra medium processing that was performed in this study, which used a methanolchloroform extraction of the medium before analysis. The difference between deconvolution and manual integration in measured concentrations for select compounds was less than $1 \%$. Therefore, batch integration with manual selection of an integral region applied to all samples simultaneously was used. The observed difference between these two methods of concentration quantification is consistent with reported best practices. ${ }^{27}$ In combination with the use of a robotic sample changer, batch-binned spectra resulted in a HTP methodology, allowing for multiple samples to be processed rapidly in a reproducible manner without the
A

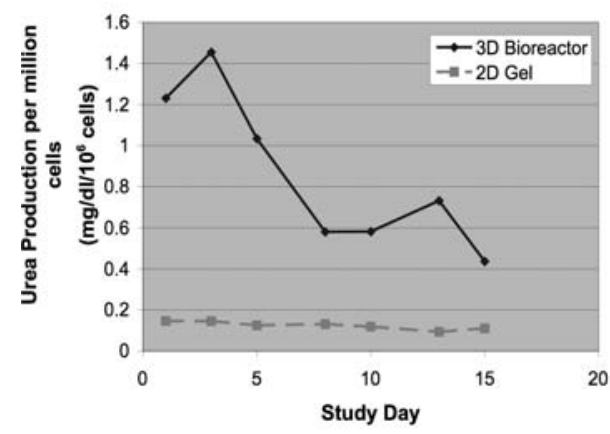

B

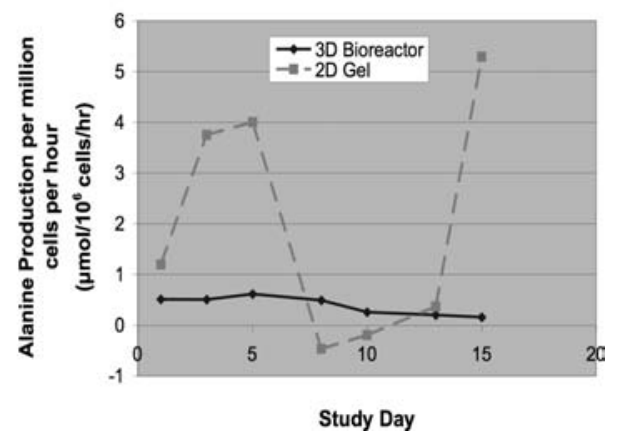

FIG. 7. Time courses of urea (A) and alanine (B) production rates in 3-dimensional (3D) bioartificial liver and 2D control cultures. 
A

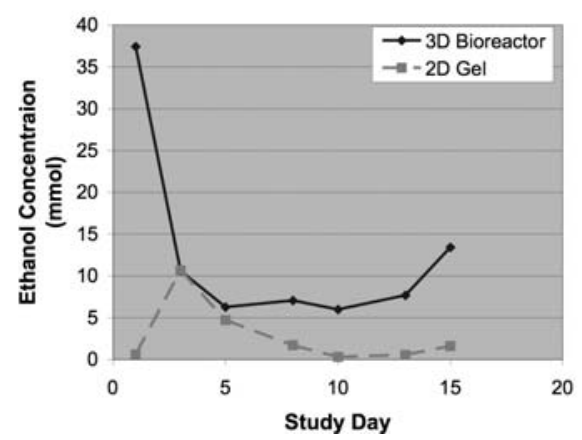

B

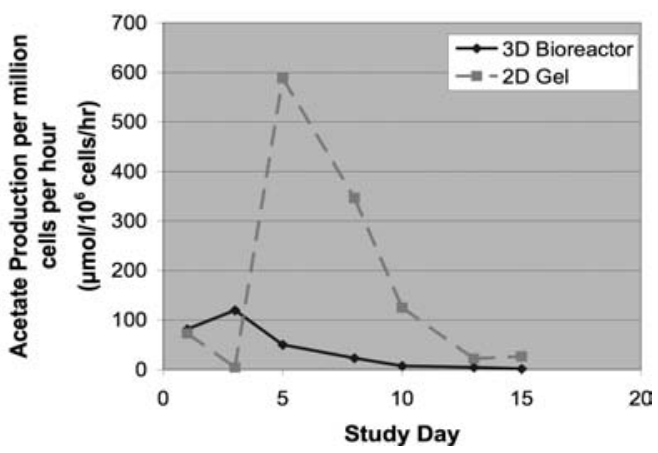

FIG. 8. Time courses of ethanol concentration $(\mathbf{A})$ and acetate production $(\mathbf{B})$ in $3 \mathrm{D}$ bioartificial liver and $2 \mathrm{D}$ control cultures.

need for intensive operator scrutiny associated with individual spectrum deconvolution methods.

A primary obstacle in tissue engineering is the inability to use the most common analytical tool of the cell biologist, the microscope, because of the opaque properties of the artificial materials used in tissue engineering. ${ }^{22,28}$ Therefore, the indirect characterization of tissue function through cell culture medium analysis is the primary means for tissue engineers to demonstrate bioartificial organ functions. The HTP ${ }^{1} \mathrm{H}$ NMR method of medium analysis results in an unbiased metabolomic "footprint," leading to well-informed tissue engineering optimization. The method permits quantitative analysis of extracellular milieu in constant contact with the cell culture, which is converted to production or consump- tion rates. Although the rates shown herein have been used for years in biochemical engineering, ${ }^{29}$ entering these data into a simple biochemical scheme is rarely done and permits easy interpretation of the complex metabolomic footprint results. This basic bioenergetic scheme of metabolism converts relative mass balance results derived from the consumption and production rates to the thickness of arrows along with the rates within the biochemical network, giving a rapid overview of carbon flow in the bioreactor, similar to other macrokinetic models. ${ }^{29,30}$

Systemic metabolic changes were evident over the time course of the experiment. In particular, Figure 9 is a biochemical schematic representation incorporating the consumption and production rates of the $2 \mathrm{D}$ and $3 \mathrm{D}$ culture
A

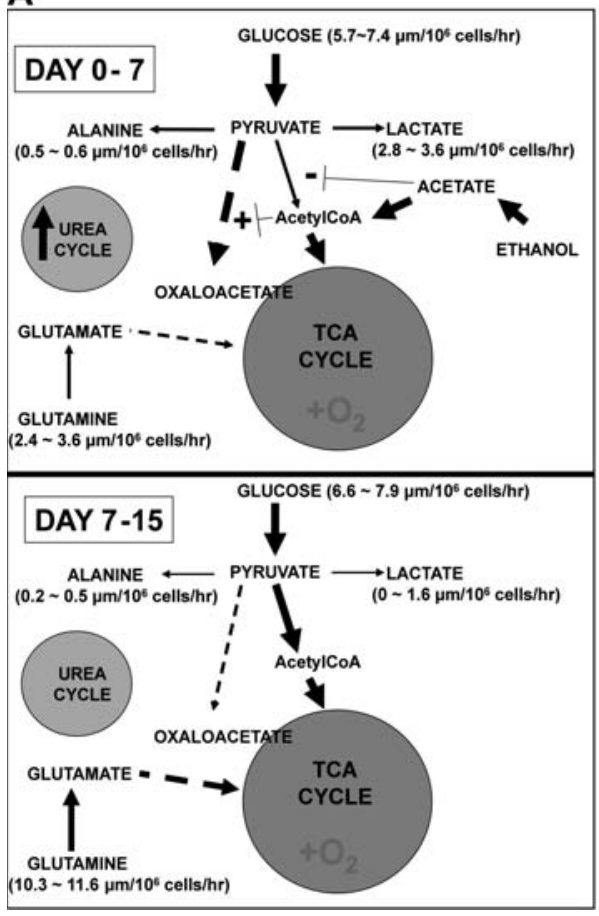

B

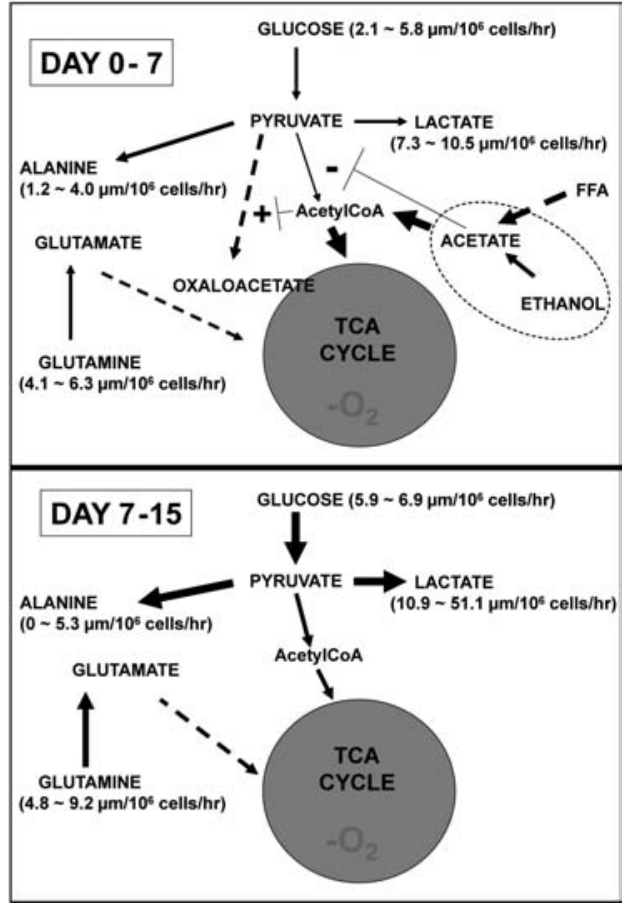

FIG. 9. Metabolic scheme for 3D bioreactor (A) and the metabolic scheme for 2D control cultures (B). 
systems across the 2-week period. Initially for the 3D BAL (Fig. 9A), the primary energy source was glucose (Fig. 6A). The bioreactor system appeared to be less reliant on glutaminolysis for energy during this initial week of culture (Fig. 6C). Glutamine is the primary carbon source for energy in transformed cells in culture. ${ }^{31}$ There was less build up of lactate, which in conjunction with glutaminolysis indicates aerobic metabolism (Fig. 6D). However, ethanol was discovered in the medium (Fig. 8A), which is likely an artifact of the initial bioreactor membrane wetting process. Therefore, this high concentration of ethanol in the medium complicates the details of metabolism.

Ethanol is known to form acetate through the combined actions of ethanol dehydrogenase and aldehyde dehydrogenase. Acetate build-up also mimicks the starved state, whereby fatty acids undergo $\beta$-oxidation, increasing the mitochondrial acetate concentration. ${ }^{25}$ High mitochondrial acetate concentrations inhibit pyruvate dehydrogenase and increase the activity of pyruvate carboxylase, resulting in glucose-derived pyruvate forming oxaloacetate rather than acetyl-CoA. ${ }^{32}$ This inhibition of pyruvate metabolism by ethanol may have been the cause of the decrease in glucose consumption during this period, because ATP, which would be sufficiently supplied by acetate metabolism, inhibits hexose kinase. ${ }^{31}$ In addition, urea production was elevated during this period (Fig. 7), indicating an abundance of ammonia present, likely due to transamination reactions. The increased glucose-derived intracellular pyruvate due to pyruvate dehydrogenase inhibition by ethanol-derived acetyl-CoA would drive the formation of alanine and could be the reason for increased alanine production. In whole animals, glucose is limited unlike the nonphysiological culture conditions inherent to ex vivo culture, and alanine becomes depleted in the liver. ${ }^{33}$ In the presence of acetate and alanine, pyruvate is shunted toward gluconeogenesis, ${ }^{33}$ but isotope tracer studies are required to confirm whether gluconeogenesis is upregulated because of elevated ethanol-derived acetate levels.

During the second phase of the 3D BAL metabolomic footprint (Fig. 9A), the primary energy sources were glucose and glutamine (Fig. 6A and C). There was no accumulation of lactate, indicating aerobic glycolysis (Fig. 6D). Ethanol and acetate had disappeared from the medium by day 7 (Fig. 8), and glucose consumption increased, which would ultimately produce intracellular pyruvate. As discussed above, without isotope tracer data, the apparent increase in glucose consumption could be real or simply a decrease in gluconeogenesis. This could explain why pyruvate remained elevated in the medium and the apparent pyruvate consumption decreased. In addition, urea production diminished during this period (Fig. 7A).

The primary energy source for the $2 \mathrm{D}$ cultures proved also to be glucose (Fig. 9B) over the first 3 time points, on days 1-5 of culturing (Fig. 6A). Lactate production during this initial phase of the study was higher than in the 3D bioreactor, which is indicative of anaerobic metabolism. Again, ethanol was discovered in the medium (Fig. 8) due possibly to the sterilization process of the culturing hood using an ethanol spray during media changes. Some medias contain ethanol-dissolved dexamethazone, but ours does not. However, more likely is that lipids accumulated as the result of cell debris in the culture dish. Metabolism of these membrane breakdown products by the surviving hepatocytes could result in high acetate levels. In addition, urea production was observed to be nominally above the level of detection during this period (Fig. 7A), although transamination reactions were active, as indicated by production of alanine (Fig. 7B). Alanine formation from pyruvate is considered to be an ammonia detoxication in the absence of the urea cycle. ${ }^{25}$

Although glucose was the principal carbon source during the second phase of the 2D culture footprint (Fig. 9B), unlike in the bioreactor, anaerobic glycolysis dominated, with massive production of lactate (Fig. 6D). Similar to the 3D BAL, ethanol and acetate concentrations diminished over time in the 2D culture (Fig. 8). Urea production was consistently low (just above detectable limits) throughout both phases of the 2D culture study (Fig. 7A), whereas alanine production, although erratic, was on average greater than that observed in the bioreactor.

In both $2 \mathrm{D}$ and $3 \mathrm{D}$ cultures other amino acids in the medium, such as valine, leucine, isoleucine, tyrosine, histidine and phenyl alanine were minor carbon sources relative to glucose and glutamine, unlike a recent study of a 3D human bioartificial liver. ${ }^{34}$

This study demonstrates the power of unbiased NMR spectroscopic analysis of culture media for metabolomic phenotyping and experimental quality control in tissue engineering. ${ }^{29}$ NMR will detect all of the most abundant metabolites in the medium with sufficient concentration. With the NMR instrumentation used in this study, $400 \mathrm{MHz}$, the limit of detection was near $100 \mu \mathrm{M}$ for a well-resolved peak. The unbiased analysis results in the discovery of unexpected metabolites, such as ethanol and acetate. Standard chromatography or spectroscopic methods would not have detected ethanol and its hepatic metabolite acetate.

Critical to the success of this type of study is the use of hormonally defined medium ${ }^{22}$ rather than medium using calf serum or other blood products as medium supplements, because such serum-based products cannot offer consistent or reliable knowledge of medium additives without additional testing. This lack of reproducible initial conditions associated with classic serum-enriched culture media prevents precise measurement of consumption and production measures from culture systems, thus illustrating the significant advantage afforded by hormonally defined media in metabolomic studies.

Metabolomic interpretation of these data can be used to improve experimental conditions, specifically, oxygen concentration of the mixed gases, microenvironmental heterogeneity, and experimental oversights. The most obvious experimental difference that resulted in metabolic effects was oxygen tension. In 3D culture systems, 95\% oxygen is used, versus $20 \%$ oxygen (i.e., air) in the 2D culture system. Quality 
assurance concerns that were identified included ethanol rinsing of the bioreactor, technician effects, and evaporation associated with the use of dry gases in the 3D BAL. Other areas of tissue culture quality monitoring this method may serve include detection of low-level contamination by yeast or bacteria, medium composition consistency, and medium component stability. Human hepatocytes cultures are precious, and this HTP ${ }^{1} \mathrm{H}$ NMR method of analyzing culture media can be used to obtain near real-time readings of metabolism, allowing for more-rapid optimization or real-time modification of long-term tissue-engineered culture conditions.

\section{ACKNOWLEDGMENTS}

Funding for the studies was provided by in part National Institutes of Health grants P30ES10126, RR-000046, GM075941-01 and CA114365-01A1.

\section{REFERENCES}

1. O'Leary DJ, Hawkes SP, Wade CG. Indirect monitoring of carbon-13 metabolism with NMR analysis of perfusate with a closed-loop flow system. Magn Reson Med 5, 572, 1987.

2. Bell J. Brown, JC, Sandler, PJ. NMR studies of body fluids. NMR Biomed 2, 242, 1989.

3. Dabos KJ, Parkinson JA, Hewage C, Nelson LJ, Sadler IH, Hayes PC, Plevris JN. H NMR spectroscopy as a tool to evaluate key metabolic functions of primary porcine hepatocytes after cryopreservation. NMR Biomed 15, 241, 2002.

4. Mendes P. Emerging bioinformatics for the metabolome. Brief Bioinform 3, 134, 2002.

5. Allen J, Hassanein, T, Bhatia, SN. Advances in bioartificial liver devices. Hepatology 34, 447, 2001.

6. Holmes E. Nicholls AW, Lindon JC, Connor SC, Connelly JC, Haselden JN, Damment SJ, Spraul M, Neidig P, Nicholson JK. Chemometric models for toxicity classification based on NMR spectra of biofluids. Chem Res Toxicol 13, 471, 2000.

7. Pham-Tuan H, Kaskavelis L, Daykin CA, Janssen HG. Method development in high-performance liquid chromatography for high-throughput profiling and metabonomic studies of biofluid samples. J Chrom B 789, 283, 2003.

8. Kell DB. Metabolomics and systems biology: making sense of the soup. Current Opin Microbiol 7, 296, 2004.

9. Allen J. Davey, HM, Broadhurst, D, Heald, JK, Rowland, JJ, Oliver, SG, Kell, DB, High-throughput classification of yeast mutants for functional genomics using metabolic footprinting. Nat Biotechnol 21, 6926, 2003.

10. Kuesel AC, Graschew G, Hull W, Lorenz W, Thielmann HW. 31P NMR studies of cultured human tumor cells. Influence of $\mathrm{pH}$ on phospholipid metabolite levels and the detection of cytidine 5'-diphosphate choline. NMR Biomed 3, 78, 1990.

11. Miccheli AT, Miccheli A, Di Clemente R, Valerio M, Coluccia P, Bizzarri M, Conti F. NMR-based metabolic profiling of human hepatoma cells in relation to cell growth by culture media analysis. Biochim Biophys Acta 1760, 1723, 2006.
12. Bubb W, Wright, LC, Cagney, M, Santangelo, RT, Sorrell, TC, Kuchel, PW. Heteronuclear NMR studies of metabolites produced by Cryptococcus neoformans in culture media: identification of possible virulence factors. Magn Reson Med 42, 442, 1999.

13. Nicholson JK, Lindon JC, Holmes E. 'Metabonomics': understanding the metabolic responses of living systems to pathophysiological stimuli via multivariate statistical analysis of biological NMR spectroscopic data. Xenobiotica 29, 1181, 1999.

14. Holmes E, Nicholson JK, Tranter G. Metabonomic characterization of genetic variations in toxicological and metabolic responses using probabilistic neural networks. Chem Res Toxicol 14, 182, 2001.

15. Gillies RJ. Nuclear magnetic resonance and its applications to physiological problems. Ann Rev Physiol 54, 733, 1992.

16. Macdonald JM, Grillo M, Schmidlin O, Tajiri DT, James TL. NMR spectroscopy and MRI investigation of a potential bioartificial liver. NMR Biomed 11, 55, 1998.

17. Kell D, Brown M, Davey HM, Dunn WB, Spasic I, Oliver SG. Metabolic footprinting and systems biology: the medium is the message. Nat Rev Micro 3, 557, 2005.

18. Kriat M, Confort-Gouny S, Vion-Dury J, Sciaky M, Viout P, Cozzone P. Quantitation of metabolites in human blood serum by proton magnetic resonance spectroscopy. A comparative study of the use of formate and TSP as concentration standards. NMR Biomed 5, 179, 1992.

19. Wolfe SP, Hsu E, Reid LM, Macdonald JM. A novel multicoaxial hollow fiber bioreactor for adherent cell types. Part 1: Hydrodynamic studies. Biotechn Bioengin 77, 83, 2002.

20. Macdonald JMWS, Roy-Chowdhury I, Kubota H, Reid LM. Effect of flow configuration and membrane characteristics on membrane fouling in a novel multicoaxial hollow-fiber bioartificial liver. Ann NY Acad Sci 944, 334, 2001.

21. Macdonald JM, Wolfe SP. Bioreactor design and process for engineering tissue from cells. U.S. Patent 6,979,308, 2005.

22. Macdonald JM, Xu A, Kubota H, LeCluyse E, Hamilton G, Liu H, Rong Y, Moss N, Lodestro C, Luntz T, Wolfe SP, Reid LM. Liver cell culture and lineage biology. In: Atala A, Lanza RP, Vacanti, J.P. eds. Methods of Tissue Engineering. Boston: Academic Press, 2002, pp. 151-195.

23. McClelland RE, Coger RN. Effects of enhanced O2 transport on hepatocytes packed within a bioartificial liver device. Tissue Eng 10, 253, 2004.

24. Gadian DG. NMR and Its Applications to Living Systems, 2nd Ed. New York: Oxford University Press 1995.

25. Lehninger AL, Nelson DL, Cox MM. Lehninger Principles of Biochemistry, 4th Ed. New York: W.H. Freeman, 2005.

26. Weljie AM, Newton J, Mercier P, Carlson E, Slupsky CM. Targeted profiling: quantitative analysis of $1 \mathrm{H}$ NMR metabolomics data. Anal Chem 78, 4430, 2006.

27. Maniara G, Cepriano A, Powell, ML. Method performance and validation for quantitative analysis by $1 \mathrm{H}$ and 31P NMR spectroscopy. Applications to analytical standards and agricultural chemicals. Anal Chem 70, 4921, 1998.

28. Macdonald JM, Griffin J, Kubota H, Griffith L, Fair J, Reid LM. Therapeutics. In: Kuhtreiber W, Lanza RP, Chick WL, eds. Cell Encapsulation Technology and Therapeutics. Boston: Birkhauser 1999. 
29. Bailey JE, Ollis DF. Biochemical Engineering Fundamentals, 2nd Ed. New York: McGraw-Hill 1986.

30. Zhou FB, Zeng A, Yuan JA. A macrokinetic model for myeloma cell culture based on stoichiometric balance. Biotechnol Appl Biochem 46, 85, 2007.

31. Mancuso A, Sharfstein SA, Fernandez EJ, Clark DS, Blanch HW. Effect of extracellular glutamine concentration on primary and secondary metabolism of a murine hybridoma: an in vivo $13 \mathrm{C}$ nuclear magnetic resonance study. Biotech Bioengin 57, 172, 1998.

32. Lieber CS. Metabolism of alcohol. Clin Liver Dis 9, 1, 2005.

33. Nicholas PC, Kim D, Crews FT, Macdonald JM. 1H NMRbased metabolomic analysis of liver, serum and brain following ethanol administration in rats. Che. Res. Tox. 21(2): 408-20, 2008.
34. Guthke R, Zeilinger K, Sickinger S, Schmidt-Heck W, Buentemeyer H, Iding K, Lehmann J, Pfaff M, Pless G, Gerlach JC. Dynamics of amino acid metabolism of primary human liver cells in 3D bioreactors. Bioprocess Biosyst. Eng. 28: 331-40, 2006.

\author{
Address reprint requests to: \\ Jeffrey M. Macdonald, Ph.D. \\ Department of Biomedical Engineering \\ University of North Carolina \\ Chapel Hill, NC 27599
}

E-mail: jmacdona@med.unc.edu 